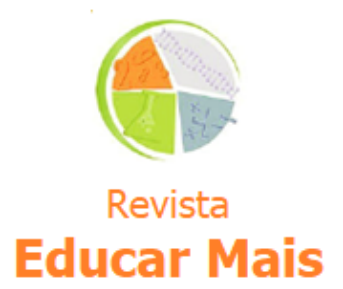

\section{Argumentos a Favor da Ação a Distância}

Arguments in favour of action at a distance.

Andre Koch Torres Assis ${ }^{1}$

\title{
RESUMO
}

Esta é uma tradução ampliada, atualizada e com novas referências do artigo "Arguments in favour of action at a distance" publicado em "Instantaneous Action at a Distance in Modern Physics - 'Pro' and 'Contra'," editado por A. E. Chubykalo, V. Pope e R. Smirnov-Rueda (Nova Science Publishers, Commack, 1999), págs. 45-56, [Ass99]. São apresentados vários argumentos a favor da ação a distância. As leis de ação a distância de Newton, Coulomb, Ampère e Weber são analisadas. São enfatizadas evidências históricas de que a eletrodinâmica de Weber chegou na propagação dos sinais eletromagnéticos deslocando-se com a velocidade da luz antes do desenvolvimento das equações de Maxwell. É discutida a implementação do princípio de Mach com a lei de Weber aplicada à gravitação.

Palavras-chave: Ação a distância; eletrodinâmica de Weber; princípio de Mach; equação de onda.

\section{ABSTRACT}

This work presents a Portuguese translation (enlarged, updated and with new references) of the paper "Arguments in favour of action at a distance" published in "Instantaneous Action at a Distance in Modern Physics - Pro and Contra", edited by A. E. Chubykalo, V. Pope and R. Smirnov-Rueda (Nova Science Publishers, Commack, 1999), pp. 45-56, [Ass99]. Several arguments in favor of instantaneous action at a distance are presented. The action at a distance laws of Newton, Coulomb, Ampère and Weber are analyzed. Historical evidence that Weber's electrodynamics led to the propagation of electromagnetic signals with light velocity prior to the development of Maxwell's equations are emphasized. The implementation of Mach's principle with Weber's law applied to gravitation is discussed.

Keywords: Action at a distance; Weber's electrodynamics; Mach's principle; wave equation.

PACS: $01.55 .+b$ (física geral - educação em física), 01.70.+w (filosofia da ciência).

\footnotetext{
${ }^{1}$ Instituto de Física 'Gleb Wataghin' Universidade Estadual de Campinas - UNICAMP - 13083-859 Campinas, São Paulo, Brasil. E-mail: assis@ifi.unicamp.br, homepage: www.ifi.unicamp.br/ assis.
} 


\section{INTRODUÇÃO}

Apresento neste artigo vários argumentos a favor da ação a distância. Mas antes vou apresentar algumas lembranças pessoais sobre este tema.

Durante o ensino médio (1977-79) aprendi a lei de Newton da gravitação (1687) e não vi nenhum problema com ela. Porém, ao ler livros sobre história da ciência descobri que Huygens e Leibniz, contemporâneos de Newton, não aceitaram suas concepções pois elas implicavam que o Sol agia diretamente sobre a Terra, a Terra agia diretamente sobre a Lua e assim por diante. Me lembro que não conseguia entender a atitude negativa de Huygens e Leibniz pois para mim era óbvio que o Sol atraía a Terra, assim como a Terra atraía a Lua e uma maçã. Naquela época eu não pensava em qualquer outro mecanismo que pudesse explicar esta atração gravitacional.

Quando estava fazendo meu curso de graduação em física (1980-83) percebi o quão problemática é a lei da gravitação de Newton devido ao seu caráter de ser uma lei de ação a distância. Por exemplo, como o Sol poderia saber a distância quanta massa há na Terra para então aplicar a força correta sobre ela? Como esta força era transmitida do Sol até a Terra? Pode um corpo de dimensões finitas agir sobre outros corpos que estão localizados em lugares nos quais o primeiro corpo não está tocando? Foi então que compreendi as críticas de Huygens e Leibniz. Nesta época também comecei a especular sobre outros mecanismos que explicassem a interação entre corpos, tal como a troca de partículas (fótons ou grávitons) deslocando-se a uma velocidade finita, a emissão de campos gravitacionais e eletromagnéticos deslocando-se com a velocidade da luz, ou então esta interação poderia ocorrer através de perturbações ocorrendo em um meio contínuo tal como um éter. Mudei então meu ponto de vista e concluí que não havia ação a distância na natureza.

Em 1986 e 1987 comecei a fazer algumas anotações sobre um assunto que designei de Princípios Gerais da Física. Ou seja, princípios que acreditava serem válidos para toda a física, incluindo a mecânica, termodinâmica, óptica ou eletromagnetismo.

O primeiro princípio era o de que as leis da física deveriam depender apenas das distâncias entre os corpos que estavam interagindo, assim como das derivadas temporais destas distâncias. Isto é, as grandezas fisicamente relevantes não deveriam depender da posição, da velocidade nem da aceleração do observador. Em vez disto, todas as leis básicas da física derivam de ter a seguinte forma: $\quad f\left(\vec{r}_{i}-\vec{r}_{j}, \vec{v}_{i}-\vec{v}_{j}, \vec{a}_{i}-\vec{a}_{j}, d^{3} \vec{r}_{i} / d t^{3}-d^{3} \vec{r}_{j} / d t^{3}, \ldots, m_{i}-m_{j}\right.$ ou $m_{i} / m_{j}, q_{i}-q_{j}$ ou $\left.q_{i} / q_{j}, \ldots\right)$. Nesta expressão $\vec{r}_{i}$ e $\vec{r}_{j}$ representam vetores que designam as posições das partículas $i$ e $j$ que possuem massas $m_{i}$ e $m_{j}$, assim como cargas elétricas $q_{i}$ e $q_{j}$, respectivamente, enquanto $\vec{v}_{i}-\vec{v}_{i}=d\left(\vec{r}_{i}-\vec{r}_{j}\right) / d t$ e assim por diante. Cheguei a este primeiro princípio geral quando descobri que na força de Lorentz, $\vec{F}=q \vec{E}+q \vec{v} \times \vec{B}$, a velocidade $\vec{v}$ era utilizada nos livros didáticos como sendo a velocidade da carga $q$ em relação ao observador, em vez de ser a velocidade da carga $q$ em relação ao imã ou em relação ao fio conduzindo uma corrente elétrica com a qual esta carga estaria interagindo. Não gostava desta interpretação que aparecia nos livros didáticos já que ela era contrária à minha intuição física. Foi este o motivo que me fez introduzir este primeiro princípio geral da física. Para mim esta velocidade $\vec{v}$ tinha de ser a velocidade entre a carga $q$ e o ímã com o qual ela estava interagindo, ou então tinha de ser a velocidade entre a carga $q$ e o fio com corrente com o qual ela estava interagindo. 
O segundo princípio geral era o de que a cinemática tinha de ser exatamente equivalente à dinâmica. Era desta maneira que eu interpretava o princípio de Mach e sua análise da experiência do balde de Newton. Não me lembro quando li pela primeira vez o livro A Ciência da Mecânica de Ernst Mach². De qualquer forma, me lembro de discutir as ideias de Mach com alguns colegas a partir de 1986. Quando o balde e a água estão em repouso em relação ao solo, a superfície da água é plana. Quando o balde e a água giram juntos em relação ao solo e em relação ao universo distante, a superfície da água é côncava. Newton pensou que esta concavidade era devida à rotação da água em relação ao espaço absoluto, sendo que para ele este espaço absoluto não tinha qualquer relação com a Terra ou com as chamadas estrelas fixas. Ernst Mach foi contra esta interpretação de Newton e acreditava que a concavidade aparecia devido à rotação relativa entre a água e as estrelas distantes. $O$ que aconteceria se o balde e a água fossem mantidos em repouso em relação ao solo, enquanto que o universo distante girasse em relação ao solo ao redor do eixo do balde, com a mesma velocidade angular que a velocidade angular da experiência original de Newton, mas na direção oposta? De acordo com a mecânica clássica a água deveria permanecer plana, enquanto que de acordo com o princípio de Mach a água deveria subir em direção às paredes do balde, assim como ocorria na experiência original de Newton. As ideias relacionais de Mach sempre pareceram mais intuitivas para mim do que as ideias absolutas de Newton baseadas no espaço vazio. Foi por este motivo que propus para mim mesmo este segundo princípio geral.

O terceiro princípio geral (escrito em Junho de 1988) foi o de que não haveria ação a distância. Nesta época eu acreditava que todas as interações entre quaisquer dois corpos deveria viajar com uma velocidade finita. Esta interação poderia ocorrer através de uma perturbação em um meio contínuo entre os dois corpos, ou através da emissão e absorção de partículas ou de campos, sendo que estas partículas ou campos viajariam com uma velocidade finita através do vácuo. Meu mecanismo preferido para a interação entre dois corpos era pela troca de partículas, tais como os grávitons.

Durante duas semanas em Fevereiro de 1985 li pela primeira vez o livro de Whittaker, Uma História das Teorias do Éter e da Eletricidade ${ }^{3}$. Contudo, nesta época a lei de ação a distância de Weber não chamou minha atenção. Foi apenas em 1988, quando estava na Inglaterra, que redescobri a lei de Weber neste livro de Whittaker e que comecei a trabalhar com ela. O motivo que me fez trabalhar com ela foi que ela satisfazia a lei de ação e reação, estava direcionada ao longo da linha reta unindo as cargas, além de depender apenas da distância entre as cargas, $r=\left|\vec{r}_{i}-\vec{r}_{j}\right|$, da velocidade radial elas, $d r / d t$, e da aceleração radial relativa entre elas, $d^{2} r / d t^{2}$. Estes aspectos compõem aquilo que chamo de uma lei de força relacional. Este era o aspecto mais marcante da força de Weber que a diferenciava da lei de força de Lorentz.

Foi também em 1988 que comecei a aplicar uma lei de Weber para a gravitação e que implementei quantitativamente com ela o princípio de Mach. Esta implementação matemática do princípio de Mach foi descrita no meu primeiro artigo, publicado em 19894. Apesar de todos estes resultados bemsucedidos, escrevi o seguinte neste artigo:

A maior limitação deste modelo é que ele está baseado em uma teoria de ação a distância. Como resultado deste fato, esta não é uma teoria final ou definitiva, mas

\footnotetext{
2 [Mac60].

3 [Whi73].

4 [Ass89].
} 
deve ser válida em sistemas com movimentos [de partículas] que variam lentamente, nos quais o retardo de tempo não representa uma limitação séria.

Hoje em dia estou mudando de ideia mais uma vez, retornando aos pontos de vista que tinha no ensino médio. Embora ainda não esteja totalmente convencido a favor da ação a distância, vejo a cada dia mais aspectos positivos relacionados com a ação a distância. Como a maioria dos físicos do século XX era contra a ação a distância, decidi escrever este artigo apresentando argumentos razoáveis em seu favor, para com isto equilibrar esta posição unilateral da maioria dos cientistas. Este artigo pode ajudar outros leitores a terem um ponto de vista mais crítico e uma mente mais aberta em relação a este tema importante.

\section{CITAÇÕES A FAVOR DA AÇÃO A DISTÂNCIA}

Apresento nesta Seção algumas citações favoráveis à ação a distância.

Ernst Mach apresentou algumas observações interessantes a este respeito em seu livro História $e$ Origem do Princípio de Conservação da Energia, publicado originalmente em 1872: ${ }^{5}$

Quais fatos valerão como fatos fundamentais, fatos sobre os quais podemos nos apoiar, isso é algo que depende do hábito e da história. Para o mais baixo nível de conhecimento, não há qualquer explicação mais suficiente do que pressão e colisão. A teoria gravitacional newtoniana inquietou, à época de seu surgimento, quase todos os pesquisadores porque ela se apoiava sobre uma ininteligibilidade inabitual. Esforçava-se por reduzir a gravitação à pressão e à colisão. Hoje a gravitação não inquieta a mais ninguém. Ela se tornou uma ininteligibilidade habitual.

É sabido que a ação a distância colocou em dificuldades muitos eminentes pensadores. "Um corpo só pode atuar onde ele está". Há, portanto, apenas pressão e colisão, mas nenhuma ação a distância. Mas onde está o corpo? Ele está apenas onde o tocamos? Vamos inverter a questão: um corpo está onde ele atua. Ele ocupa um pequeno espaço para o tato, um maior para a audição, um ainda maior para a visão. Como é que o tato chega a nos ditar sozinho onde um corpo está? Além disso, a ação por contato pode ser considerada um caso especial da ação a distância.

Burniston Brown apresentou alguns comentários interessantes na introdução de seu livro sobre ação a distância: ${ }^{6}$

Quando o autor [a saber, o próprio Burniston Brown] estava tentando escrever um livro sobre o método científico ele teve de encarar, quase que imediatamente, um problema sério. O motivo para esta dificuldade foi que, para explicar o método científico, tem de ser feita uma definição clara entre fatos e teorias. As teorias são inventadas para fornecer uma explicação causal aos fatos. Para minha surpresa, embora a palavra fato seja usada a todo momento por todas as pessoas, ninguém sabia como definir seu significado - cientistas, advogados, filósofos - ninguém sabia. Bertrand Russell disse que um fato era "alguma coisa que tornava verdadeira uma proposição" - contudo, a questão é, o que é esta 'alguma coisa'? (sem mencionar o que se entende por 'verdade'). Após dezoito meses de pensamento cuidadoso decidi que:

Um fato é uma afirmação que pode ser verificada.

Muitos anos depois percebi que ação a distância não é apenas uma outra teoria de propagação da força tal como a propagação balística, ou ondas propagando-se em

\footnotetext{
5 [Mac11, pág. 56] e [Mac14, págs. 70-71].

6 [Bro82, pág. 1]
} 
um éter. Decidi apresentar este ponto de vista em uma palestra dada em Oxford ao mostrar o efeito de um ímã atuando sobre um outro ímã suspenso [por um fio]. Então assinalei que ocorria uma ação observável em uma distância observável, de tal forma que se qualquer membro da audiência afirmasse que não havia ação a distância, era esta pessoa que estava fazendo hipóteses. Ninguém tentou negar o que afirmei. Por qual motivo não devemos admitir que, algumas vezes, o que aparenta estar acontecendo está de fato acontecendo?

A recusa em aceitar a ação a distância, levou a todas as dificuldades e às explicações tortuosas conectadas com vórtices de éter, ondas, espaço-tempo curvo, assim como muitas outras explicações - juntamente com esforços experimentais malogrados para detectar o éter.

O tempo chegou para cortar o nó górdio ao abolir todos os éteres, abandonar a atribuição de propriedades físicas ao vazio (tal como $\varepsilon_{0}$ e $\mu_{0}$ ), e rejeitar construções puramente matemáticas tais como o espaço-tempo.

Para muitas outras citações e discussões importantes indico ao leitor o livro muito relevante de Graneau e Graneau, Newton Contra Einstein - Como a Matéria Interage com a Matéria’. Este grande livro discute os dois mecanismos principais que já foram propostos na história da ciência para explicar a interação da matéria, a saber, ação a distância e ação por contato.

\section{AS LEIS DE FORÇAS FUNDAMENTAIS QUE ATUAM POR AÇÃO A DISTÂNCIA}

Apresento nesta Seção as principais leis de ação a distância que já foram propostas na física.

Todas as fórmulas estão no Sistema Internacional de Unidades MKSA. Para referências e citações sobre os trabalhos originais, ver nossos livros ${ }^{8}$. A força de ação a distância mais antiga e importante é a lei da gravitação de Newton (1687). Na notação vetorial moderna e força exercida pela massa gravitacional $m_{g 1}$ sobre a massa gravitacional $m_{g 2}$ pode ser escrita como:

$$
\vec{F}=-G m_{g 1} m_{g 2} \frac{\hat{r}}{r^{2}}
$$

Aqui $G=6,67 \times 10^{-11} \mathrm{Nm}^{2} / \mathrm{kg}^{2}$ é a constante gravitacional, $r$ é a distância entre as partículas, sendo $\hat{r}$ o vetor unitário que aponta ao longo da reta que conecta as duas partículas.

Cem anos depois Coulomb chegou na força entre as cargas elétricas $q_{1}$ e $q_{2}$, a saber:

$$
\vec{F}=\frac{q_{1} q_{2}}{4 \pi \varepsilon_{0}} \frac{\hat{r}}{r^{2}}
$$

Aqui $\varepsilon_{0}=8,85 \times 10^{-12} C^{2} N^{-1} m^{-2}$ é a constante chamada de permissividade do vácuo. Esta força é muito similar à lei da gravitação de Newton.

Na mesma época Coulomb chegou na força entre dois polos magnéticos pontuais, $q_{1}^{p m}$ e $q_{2}^{p m}$, como sendo dado por:

\footnotetext{
7 [GG93].

8 [Ass94], [Cha09], [AC11], [Ass13], [Ass14a], [AC15] e [Ass15].
} 


$$
\vec{F}=\frac{\mu_{0}}{4 \pi} q_{1}^{p m} q_{2}^{p m} \frac{\hat{r}}{r^{2}}
$$

Aqui $\mu_{0}=4 \pi \times 10^{-7} \mathrm{kgmC}^{-2}$ é a constante chamada de permeabilidade magnética do vácuo.

Entre 1820 e 1826 Ampère chegou na força entre os elementos de corrente $I_{1} d \vec{l}_{1}$ e $I_{2} d \vec{l}_{2}$ como sendo dada por:

$$
d^{2} \vec{F}=-\frac{\mu_{0}}{4 \pi} I_{1} I_{2} \frac{\hat{r}}{r^{2}}\left[2\left(d \vec{l}_{1} \cdot d \vec{l}_{2}\right)-3\left(\hat{r} \cdot d \vec{l}_{1}\right)\left(\hat{r} \cdot d \vec{l}_{2}\right)\right]
$$

Ao integrar esta expressão ao longo dos dois circuitos fechados $C_{1}$ e $C_{2}$ ele chegou no seguinte resultado:

$$
\vec{F}=-\frac{\mu_{0}}{4 \pi} I_{1} I_{2} \oint_{C_{1}} \oint_{C_{2}} \frac{\hat{r}}{r^{2}}\left(d \vec{l}_{1} \cdot d \vec{l}_{2}\right)
$$

Com o objetivo de unificar as leis de Coulomb, Ampère e Faraday (1831) Weber propôs em 1846 a seguinte força entre duas cargas elétricas pontuais $q_{1}$ e $q_{2}$ :

$$
\vec{F}=\frac{q_{1} q_{2}}{4 \pi \varepsilon_{0}} \frac{\hat{r}}{r^{2}}\left(1-\frac{\dot{r}^{2}}{2 c^{2}}+\frac{r \ddot{r}}{c^{2}}\right)
$$

Nesta expressão $\dot{r}=\frac{d r}{d t}, \ddot{r}=\frac{d^{2} r}{d t^{2}}$ e $c=1 / \sqrt{\mu_{0} \varepsilon_{0}}$.

Todas estas expressões possuem a seguinte propriedade básica da ação a distância. Suponha que uma das partículas está localizada no Sol e a outra na Terra. Estas leis afirmam que se deslocarmos uma das partículas, aumentando sua distância em relação à outra partícula, a força sobre esta segunda partícula vai mudar instantaneamente.

\section{ARGUMENTOS FAVORÁVEIS À AÇÃO A DISTÂNCIA}

Todas estas leis de força satisfazem o princípio de ação e reação. Este fato significa conservação do momento linear para qualquer sistema de partículas que estão interagindo de acordo com estas leis. Estas forças também estão ao longo da linha reta que conecta as partículas, sendo que este fato significa conservação do momento angular. Estas forças também podem ser deduzidas de energias potenciais, sendo que este fato significa conservação da energia. Estes três aspectos são muito importantes de um ponto de vista conceitual e também simplificam enormemente os cálculos.

Usualmente as pessoas que são contrárias à ação a distância tentam explicar a interação entre cargas elétricas por meio da pressão e das colisões. Isto é, cada carga deve emitir corpúsculos ou campos que irão deslocar-se no espaço com uma velocidade finita, afetando a segunda carga quando estes corpúsculos ou campos chegam nela.

Hoje em dia existe o mecanismo inverso. Isto é, utilizando as forças de longo alcance de Newton e de Coulomb é possível explicar a experiência de espalhamento de Rutherford (que pode ser 
considerada análoga a uma colisão entre duas bolas de bilhar) sem que os dois corpos que estão interagindo cheguem a se tocar.

Um dos argumentos mais fortes a favor da ação a distância é o fato de quão poderosa ela pode ser. Mostro aqui como várias coisas podem ser deduzidas começando apenas com a força de Weber entre cargas pontuais, equação (6). Para discussões e referências detalhadas veja as obras de Assis, Bueno, Chaib e Hernandes ${ }^{9}$. Se não há movimento entre as cargas, reobtemos a força de Coulomb, a partir da qual a lei de Gauss também pode ser deduzida. Logo, se forem fornecidas as propriedades dielétricas dos materiais obtidas experimentalmente, toda a eletrostática pode ser deduzida a partir da lei de Weber. Quando existem cargas em movimento, como no caso da interação entre condutores conduzindo correntes elétricas, a força de Weber vai fornecer a força de Ampère entre elementos de corrente, sendo que esta força de Ampère prevê corretamente a força entre condutores de tamanhos finitos. Esta força de Ampère também dá origem à lei circuital magnética e à lei de não existência dos monopolos magnéticos. Quando temos correntes variáveis no tempo, ou então condutores móveis conduzindo correntes elétricas, a força de Weber também leva à lei de indução de Faraday. Mostro a seguir como Weber e Kirchhoff deduziram a propagação de sinais eletromagnéticos propagando-se com a velocidade da luz antes que Maxwell tivesse escrito suas equações do eletromagnetismo. É fascinante observar que todos estes resultados profundos podem ser deduzidos de uma lei tão simples quanto a força de Weber entre cargas pontuais.

Quando a lei de Weber para a gravitação é combinada com o princípio de equilíbrio dinâmico, tornase possível implementar quantitativamente o princípio de $\mathrm{Mach}^{10}$. Ou seja, a inércia de qualquer corpo é deduzida como sendo devida a uma interação gravitacional deste corpo com os corpos distantes do universo. Quando falo de inércia aqui estou me referindo à massa inercial $m_{i}$ de qualquer corpo, assim como estou me referindo a outras coisas relacionadas com esta massa inercial, tal como a energia cinética $T=m_{i} v^{2} / 2$, o momento linear, o momento angular e as forças inerciais (tais como o termo $m_{i} \vec{a}$ que aparece na segunda lei de Newton, a força centrífuga e a força de Coriolis). Isto é, de acordo com a mecânica relacional a inércia de qualquer corpo é entendida como sendo sua resistência a ser acelerada em relação aos corpos materiais distantes. Novamente todos estes resultados são obtidos utilizando a lei de ação a distância de Weber, sem utilizar qualquer retardo de tempo. Também a precessão do periélio dos planetas é deduzida corretamente da lei de Weber. Considero a implementação matemática do princípio de Mach como sendo o resultado mais poderoso já obtido com a lei de Weber aplicada à gravitação.

\section{PROPAGAÇÃo DE SINAIS ELETROMAGNÉTICOS}

Em geral afirma-se que a propagação de sinais eletromagnéticos tal como pelas antenas prova que a ação a distância está errada. Discuto este tópico nesta Seção.

A primeira coisa que deve ser mencionada é que a grandeza eletromagnética $c=1 / \sqrt{\mu_{0} \varepsilon_{0}}$ (a razão da unidade eletromagnética de carga para a unidade eletrostática de carga) foi introduzida na física na força de Weber de $1846^{11}$. Neste ano de 1846 ele introduziu uma constante $a$, que foi chamada

\footnotetext{
${ }^{9}$ [Ass94], [BA01], [AH07], [Cha09], [AH09], [AC11], [AH13], [AC15], [BA15] e [Ass15].

10 [Ass89], [Ass93], [Ass95], [Ass98], [Ass13] e [Ass14a].

11 [Web46] com tradução para a língua inglesa em [Web07].
} 
por ele como $a=4 / c$ em 1856, sendo que esta grandeza $c$ como introduzida originalmente por Weber é a razão da unidade eletrodinâmica de carga para a unidade eletrostática de carga. Contudo, a constante $c=4 / a$ de Weber não é a mesma coisa que a constante atual também representada pela letra $c$, mas sendo dada por $c=1 / \sqrt{\mu_{0} \varepsilon_{0}}$. A constante original $c$ de Weber é dada por $\sqrt{2}$ vezes a constante $c$ atual dada por $c=1 / \sqrt{\mu_{0} \varepsilon_{0}}$. Os primeiros a medir esta constante foram Weber e Kohlrausch em um trabalho conjunto de 1856, no qual encontraram (usando aqui a grandeza $c$ com seu significado atual): $\sqrt{2} c=4,39 \times 10^{8} \mathrm{~m} / \mathrm{s}$, de tal forma que $c=3,1 \times 10^{8} \mathrm{~m} / \mathrm{s}$. Este resultado mostrou uma das primeiras conexões quantitativas entre o eletromagnetismo e a óptica.

Mas o que gostaria de enfatizar aqui é o trabalho de Gustav Kirchhoff que chegou na equação do telegrafista em 1857 ao trabalhar com a teoria de ação a distância de Weber. Ele publicou três artigos principais relacionados diretamente com este tema, um trabalho em 1850 e dois em 1857, sendo que todos eles já encontram-se traduzidos para a língua inglesa ${ }^{12}$. O artigo de Kirchhoff foi publicado nos Anais editados por Poggendorff, hoje em dia conhecidos como Annalen der Physik. Poggendorff escreveu uma nota após o artigo de Kirchhoff de 1857 informando que após ter visto este artigo de Kirchhoff se encontrou com Weber em Berlim. Weber então lhe mostrou essencialmente os mesmos resultados que Kirchhoff havia obtido. Mas Weber ainda não havia enviado seu trabalho para publicação pois estava esperando os resultados de algumas experiências sobre este tema, experiências estas que Weber estava realizando juntamente com R. Kohlrausch ${ }^{13}$. $O$ trabalho simultâneo e mais completo de Weber só foi publicado em $1864^{14}$. Weber e Kirchhoff trabalharam de forma independente um do outro e previram a existência de modos periódicos de oscilação da corrente elétrica propagando-se com a velocidade da luz em um circuito condutor com resistência desprezível.

Em seu primeiro artigo de 1857 Kirchhoff considerou um fio condutor curvo de forma genérica, com seção reta circular, que poderia estar aberto ou fechado. Escreveu então uma lei de Ohm generalizada levando em conta as cargas livres espalhadas ao longo da superfície do fio, assim como a indução devida à variação da intensidade da corrente em todas as partes do fio, a saber:

$$
\vec{J}=-g\left(\nabla \emptyset+\frac{\partial \vec{A}}{\partial t}\right)
$$

Nesta equação $\vec{J}$ representa a densidade de corrente, $g$ a condutividade do fio, $\varnothing$ é o potencial elétrico, sendo $\vec{A}$ a grandeza chamada hoje em dia de vetor magnético potencial. Ele calculou o potencial $\varnothing$ ao integrar o efeito de todas as cargas distribuídas na superfície do fio:

$$
\emptyset(x, y, z, t)=\frac{1}{4 \pi \varepsilon_{0}} \iint \frac{\sigma\left(x^{\prime}, y^{\prime}, z^{\prime}\right) d a^{\prime}}{\left|\vec{r}-\vec{r}^{\prime}\right|} .
$$

Aqui $\vec{r}=x \hat{x}+y \hat{y}+z \hat{z}$ representa o ponto no qual o potencial está sendo calculado, $t$ representa 0 tempo, enquanto que $\sigma$ é a densidade superficial de carga. Após realizar esta integração por toda a superfície do fio de comprimento $l$ e raio $\alpha$ chegou ao seguinte resultado:

\footnotetext{
12 [Kir49], [Kir50], [Kir57b], [Kir57a], [Kir57c] e [GA94].

13 [Pog57].

14 [Web64].
} 


$$
\emptyset(s, t)=\frac{\alpha \sigma(s, t)}{\varepsilon_{0}} \ln \frac{l}{\alpha}
$$

sendo $s$ uma distância variável ao longo do fio medida a partir de uma origem fixa.

Obteve o vetor potencial $\vec{A}$ partindo da força de Weber, isto é:

$$
\vec{A}(x, y, z, t)=\frac{\mu_{0}}{4 \pi} \iiint\left[\vec{J}\left(x^{\prime}, y^{\prime}, z^{\prime}, t\right) \cdot\left(\vec{r}-\vec{r}^{\prime}\right)\right]\left(\vec{r}-\vec{r}^{\prime}\right) \frac{d x^{\prime} d y^{\prime} d z^{\prime}}{\left|\vec{r}-\vec{r}^{\prime}\right|^{5}} .
$$

Nesta equação a integração é para ser feita ao longo do volume do fio.

Após realizar esta integração chegou no seguinte resultado:

$$
\vec{A}(s, t)=\frac{\mu_{0}}{2 \pi} I(s, t) \ln \frac{l}{\alpha} \hat{\alpha} .
$$

Nesta equação a grandeza $I(s, t)$ representa a intensidade da corrente que pode variar não apenas ao longo do comprimento $s$ do fio, mas também ao longo do tempo $t$.

Considerando que $I=J \pi \alpha^{2}$ e que a resistência $R$ do fio é dada por $R=\ell /\left(\pi g \alpha^{2}\right)$, a componente longitudinal da lei de Ohm foi então escrita da seguinte forma:

$$
\frac{\partial \sigma}{\partial s}+\frac{1}{2 \pi \alpha} \frac{1}{c^{2}} \frac{\partial I}{\partial t}=-\frac{\varepsilon_{0} R}{\alpha l \ln (l / \alpha)} I
$$

Para relacionar as duas grandezas desconhecidas, $\sigma$ e $I$, Kirchhoff utilizou a equação para a conservação de cargas. Esta equação foi escrita da seguinte forma:

$$
\frac{\partial I}{\partial s}=-2 \pi \alpha \frac{\partial \sigma}{\partial t}
$$

Utilizando as equações (12) e (13) obtém-se então a equação do telegrafista, a saber:

$$
\frac{\partial^{2} \xi}{\partial s^{2}}-\frac{1}{c^{2}} \frac{\partial^{2} \xi}{\partial t^{2}}=\frac{2 \pi \varepsilon_{0} R}{l \ln (l / \alpha)} \frac{\partial \xi}{\partial t}
$$

Nesta equação a grandeza $\xi$ pode representar qualquer uma das seguintes grandezas: $I, \sigma, \varphi$ ou a componente longitudinal do potencial vetor $\vec{A}$.

Quando a resistência do fio é desprezível, esta equação prevê a propagação de sinais ao longo do fio deslocando-se com a velocidade da luz.

Embora nesta dedução tenha sido utilizada a interação entre qualquer par de cargas como sendo dada pela lei de ação a distância de Weber, o efeito coletivo da perturbação propaga-se com a velocidade da luz ao longo do fio.

Este resultado tem uma certa similaridade com a propagação das ondas sonoras deduzida por Newton e também com a propagação de sinais ao longo de uma corda esticada obtida por d'Alembert. Em todos estes casos foi utilizada a mecânica clássica newtoniana, sem ter sido introduzido o tempo 
retardado, sem ter sido introduzida a corrente de deslocamento, e sem ter sido utilizado qualquer campo deslocando-se com a velocidade da luz. Embora a interação entre quaisquer duas partículas em todos estes casos (sinal eletromagnético no fio, som no ar ou onda em uma corda) tenha sido do tipo de ação a distância, o comportamento coletivo do sinal ou da perturbação viaja com uma velocidade finita.

Nestes três casos temos um sistema de muitos corpos (moléculas no ar, moléculas na corda e partículas eletrizadas no fio) no qual as partículas possuem inércia. Será possível deduzir a propagação de sinais eletromagnéticos no vácuo, tal como ocorre na comunicação por rádio, utilizando uma teoria de ação a distância? Acredito que a resposta a esta questão seja positiva. Na prática não existe um sistema composto apenas de duas partículas. Em qualquer antena existem muitas partículas eletrizadas. Mesmo que seja removido qualquer meio material entre duas antenas, ainda haverá um gás de fótons entre elas. A ação a distância ocorrendo não apenas entre as cargas das duas antenas, mas também entre as cargas de cada antena e este gás de fótons localizado na região entre as antenas, pode dar origem a um comportamento coletivo que é chamado de radiação eletromagnética e que se propaga com a velocidade da luz. Além do mais, pelo princípio de Mach os corpos localizados no universo distante sempre precisam ser levados em consideração em todos os casos. Afinal de contas, as propriedades inerciais de qualquer partícula eletrizada surgem de sua interação gravitacional com a matéria distante do $\operatorname{cosmos}^{15}$. Estou trabalhando no momento neste assunto de antenas utilizando a eletrodinâmica de Weber. Estou ampliando a análise de Kirchhoff para considerar os casos de guias de onda, cabos coaxiais, antenas de dipolo e outras situações que lidam com circuitos eletromagnéticos mecânicos abertos.

Deve ser enfatizado que os trabalhos de Weber e Kirchhoff de 1856-57 foram publicados antes de Maxwell escrever suas equações em 1861-64. Quando Maxwell introduziu a corrente de deslocamento $\left(1 / c^{2}\right) \partial \vec{E} / \partial t$ ele estava utilizando a constante $c$ introduzida por Weber em 1846. Ele também estava ciente da medida de 1855-57 desta constante feita por Weber e Kohlrausch, que encontraram que ela tinha o mesmo valor que a velocidade da luz. Maxwell também conhecia a dedução da equação do telegrafista feita por Weber e Kirchhoff na qual obtiveram a propagação dos sinais eletromagnéticos deslocando-se com a velocidade da luz.

\section{PROBLEMAS COM A AÇÃO POR CONTATO}

Além de apresentar aspectos positivos da ação a distância, discuto aqui alguns problemas que existem na ação por contato. Nesta expressão "ação por contato" incluo todos os tipos de mecanismo que já foram propostos para explicar a interação entre corpos sem utilizar a ação a distância instantânea. Esta ação por contato pode ocorrer pela troca de partículas (fótons e grávitons virtuais), pela propagação de campos contínuos eletromagnéticos e gravitacionais, pela perturbação de um éter, através da ação a distância retardada, etc.

No eletromagnetismo clássico existem usualmente os potenciais de Liénard-Wiechert baseados no tempo retardado. Problemas com este enfoque foram apontados por Chubykalo e Smirnov-Rueda ${ }^{16}$ e por Whitney ${ }^{17}$.

\footnotetext{
15 [Ass13] e [Ass14a].

16 [CSR96], [CSR97] e [CSR98].

17 [Whi91] e [Whi92].
} 
Usualmente supõe-se que a gravidade propaga-se na velocidade da luz. Contudo, em um artigo muito interessante discutindo este tópico, Van Flandern mostrou que esta velocidade nunca foi observada diretamente. Além disso, muitos argumentos teóricos e medidas experimentais indicam que se esta velocidade for finita, então ela deve ser muitas ordens de grandeza maior do que a velocidade da luz $c^{18}$. Em particular, embora exista o fenômeno da aberração para a luz, não existe o fenômeno da aberração para a gravidade. Em relação a este aspecto, gostaria de apontar a discussão de Pope e Osborne da ação a distância relacionada com a gravitação e com a inércia ${ }^{19}$.

É satisfatório para a mente de muitas pessoas (embora não para mim) falar de mecanismos para as interações entre corpos como sendo devidos a uma troca de partículas ou de campos, falar da conexão entre os corpos (tal como a conexão de dois corpos através de um meio como o éter entre eles), etc. Eu próprio, por outro lado, não consigo formar imagens mentais destes campos abstratos ou destas entidades abstratas. Existem também outros problemas com a ação por contato. Se um ímã no formato de barra desloca-se em relação ao laboratório com uma velocidade constante de $1 \mathrm{~m} / \mathrm{s}$, será que o campo magnético devido a este ímã também vai se deslocar em relação ao laboratório com esta velocidade de $1 \mathrm{~m} / \mathrm{s}$ ? Ou será que este campo magnético vai deslocar-se com a velocidade da luz? Ou será que este campo magnético não vai deslocar-se em relação ao laboratório, mas vai apenas gerar um campo elétrico que também será estacionário em relação à Terra? As mesmas questões podem ser colocadas para os campos elétricos e magnéticos devidos a uma carga pontual deslocandose em relação ao laboratório.

Como pode uma coisa imaterial, tal como um campo eletromagnético, interagir com partículas materiais eletrizadas? As pessoas que não gostam de ação a distância preferem pensar nas interações como ocorrendo através de uma pressão ou da ação por contato. Por este motivo estas pessoas postulam que cada carga elétrica emite pequenas partículas (tais como fótons virtuais ou então outros tipos de corpúsculos) de tal forma que esta primeira carga só vai interagir com uma segunda carga quando estas partículas emitidas pela primeira carga chegarem na segunda carga. Algumas outras pessoas postulam que a primeira carga emite um campo eletromagnético que vai propagar-se no espaço e apenas quando este campo eletromagnético alcançar a segunda carga é que esta segunda carga vai sentir ou ser afetada pela primeira carga. Contudo, estas pessoas não especificam como vai ocorrer esta interação entre a segunda carga e estas supostas pequenas partículas (ou campos eletromagnéticos) emitidas pela primeira carga.

Além destes fatos negativos com a ação por contato, um outro aspecto muito importante da ação a distância é que ela elimina grandezas ou conceitos que não são observados. Existe uma regra simples na física que é sempre esquecida pela maioria das pessoas. Como os campos eletromagnéticos, os fótons virtuais, os grávitons, o éter e as outras grandezas mentais não são observadas diretamente, deveria ser evitado a utilização destes conceitos virtuais na física. O que se observa de fato é o movimento de corpos materiais, tais como a maçã aproximando-se da Terra, um ímã influenciando o movimento de um outro ímã, um corpo eletrizado influenciando o movimento de um outro corpo eletrizado, etc. Como existem teorias que lidam apenas com estas entidades observadas, tais como a teoria da gravitação de Newton e a teoria eletrodinâmica de Weber, estas teorias deveriam ser preferidas no lugar de outras teorias que utilizam concepções fictícias e que não são empíricas.

\footnotetext{
18 [Van97].

19 [P095].
} 
Listarei aqui algumas destas entidades mentais (ou conceitos fictícios de pensamento) que já foram postuladas para explicar a gravitação (pode ser feita uma lista semelhante para as interações eletromagnéticas): (I) Descartes propôs em 1644 que a gravidade era devida a um vórtice de uma matéria sutil circulando ao redor o eixo Norte-Sul da Terra. Huygens seguiu este enfoque, mas assumiu que pequenas partículas circulavam ao redor da Terra em todas as direções e não apenas ao redor de um eixo. (II) O próprio Newton especulou sobre um outro mecanismo que apresentou na Questão 21 de seu livro Óptica. Para ele a gravidade poderia ser devida a um meio que preencheria todo o espaço, mudando sua densidade em função da distância até o centro dos corpos (sendo mais rarefeito dentro dos corpos e mais denso fora deles). Os corpos macroscópicos seriam então impelidos a deslocar-se das partes mais densas para as partes mais rarefeitas deste meio devido a uma força elástica exercida por este meio ${ }^{20}$. Este meio proposto por Newton estava essencialmente parado e não circulava ao redor dos corpos. (III) Cem anos mais tarde o cientista Le Sage propôs uma ideia diferente. Para ele todo o espaço estaria preenchido de partículas minúsculas deslocando-se em todas as direções com grandes velocidades. Dois corpos grandes que estivessem próximos entre si iriam se proteger do bombardeio destes corpúsculos, como se fossem escudos um para o outro (tal como se estivessem fazendo sombras para a luz). Desta forma cada um destes dois corpos receberia, do lado que estivesse de frente para o outro corpo, menos corpúsculos do que os corpúsculos que receberia do outro lado. Como uma consequência deste bombardeamento desigual que ocorreria dos dois lados de cada corpo, cada um deles iria deslocar-se em direção ao outro corpo, como se estivesse sobre a ação de uma força variando do tipo $1 / r^{2}$. (IV) No final do século XIX, após os trabalhos de Faraday e Maxwell baseados no conceito de campos, as pessoas começaram a falar de um campo gravitacional contínuo gerado pelos corpos e propagando-se no espaço com uma velocidade finita. Um corpo de prova (também chamado de corpo de teste) não interagiria diretamente com outros corpos, interagindo apenas com o campo gravitacional que existisse na posição em que este corpo está localizado, sendo este campo emitido pelos outros corpos. (V) Em vez de pensar em termos de um campo gravitacional contínuo, algumas pessoas imaginam que cada corpo emite corpúsculos chamados de grávitons que deslocam-se pelo espaço com uma velocidade finita (considerada usualmente como sendo a velocidade da luz). Quando estes grávitons emitidos pelo corpo $A$ colidissem com outros corpos $B, C, D, \ldots$, então estes outros corpos $B, C, D, \ldots$, deslocariam- se em direção ao corpo $A$. (VI) Uma outra entidade fictícia original (conceito mental ou conceito de pensamento) foi proposta em 1916 com a teoria da relatividade geral de Einstein, a saber, o espaço curvo. De acordo com Einstein, um corpo não emite corpúsculos voadores (tais como balas de revólver). O corpo vai apenas curvar o espaço ao seu redor. Um corpo de prova iria então deslocarse em direção ao primeiro corpo devido à curvatura local do espaço na região em que se encontra, sendo que este deslocamento não seria mais ocasionado por uma interação direta e ocorrendo à distância.

Poderiam ser citadas aqui muitas outras entidades fictícias ou de pensamento que foram inventadas para explicar a gravidade. Contudo, estes seis exemplos já são suficientes para ilustrar o que queremos dizer. Em vez de postular todos estes conceitos (o vórtice de Descartes, o éter de Newton, os corpúsculos de Le Sage, o espaço curvo de Einstein, etc.), é muito mais simples considerar apenas aquilo que de fato é observado na natureza. Ou seja, considerar apenas a maçã, a Terra e o espaço vazio entre estes dois corpos. A lei de Newton da gravitação lida apenas com estas grandezas (massa do corpo A, massa do corpo B e a distância entre eles), explicando os fatos observados (tal como a

20 [New79, págs. 350-352] e [New96, págs. 258-259]. 
aceleração de queda livre da maçã em direção à Terra). Por este motivo a lei de Newton deve ser preferida em relação a todas as outras explicações que utilizam entidades fictícias.

Considero agora o magnetismo. Os fenômenos magnéticos são usualmente explicados em termos de um campo magnético $\vec{B}$. Este campo magnético é obtido pela regra da mão direita. Dois exemplos: (A) Se um circuito circular está localizado no plano $x y$, centrado na origem, com uma corrente elétrica fluindo no sentido horário, então o campo magnético no centro do círculo vai apontar na direção negativa do eixo $z$, isto é, na direção do vetor unitário $-\hat{z}$. (B) Considere agora um circuito retilíneo ao longo do eixo $z$ com a corrente elétrica fluindo na direção positiva do eixo $z$. Neste caso o campo magnético em qualquer ponto fora do eixo $z$ vai apontar ao longo da direção poloidal $\hat{\varphi}$, formando planos ortogonais ao eixo $z$. Estes dois exemplos violam o princípio da razão suficiente de Leibniz. De acordo com este princípio, há sempre um motivo para qualquer coisa que aconteça. Ele também pode ser formulado de outra maneira, a saber, nada acontece sem que exista uma razão pela qual este acontecimento tenha de ser assim e não possa ser de outra maneira. O princípio da razão suficiente de Leibniz também é um princípio de simetria. Vejamos o primeiro exemplo, caso (A). Como a corrente está fluindo no plano $x y$, todos os efeitos que ocorrem neste plano têm de estar localizados neste plano. Por exemplo, o campo magnético no centro do círculo não tem um motivo para escolher o sentido negativo do eixo $z$ em vez de escolher o sentido positivo do eixo $z$. Logo, como não há um motivo suficiente que diferencie o lado positivo do lado negativo do eixo $z$, o campo magnético não pode fazer esta escolha. Ou seja, ele não pode optar para apontar no sentido negativo $-\hat{z}$. Vejamos agora o segundo exemplo, caso (B). Qualquer ponto $(x, y)$ localizado fora do eixo $z$ forma um plano com a corrente retilínea que está fluindo ao longo do eixo $z$. Logo, o campo magnético, a força magnética ou qualquer outra entidade física que possa existir neste ponto $(x, y)$ também tem de estar localizada neste plano. Afinal de contas, não há qualquer razão suficiente que faça com que esta entidade escolha apontar na direção poloidal positiva $+\hat{\varphi}$ em vez de apontar na direção poloidal negativa $-\hat{\varphi}$. Ou seja, como não existe um motivo suficiente pelo qual o campo magnético possa escolher a regra da mão direita em vez da regra da mão esquerda, ele não pode fazer esta escolha. A análise apresentada aqui leva à conclusão de que não existe o campo magnético descrito em todos os livros didáticos. Toda esta discussão mostra quão problemático é o conceito de campo magnético quando considerado de um ponto de vista filosófico ou em termos dos princípios mais básicos de simetria da física.

Deve ser observado que na força magnética de Lorentz que age sobre uma carga pontual ou sobre um elemento de corrente existe um produto vetorial duplo. Outra maneira de fazer esta afirmação é observar que para obter a força magnética no eletromagnetismo clássico temos de utilizar a regra da mão direita duas vezes. Um produto vetorial (ou uma regra da mão direita) aparece na expressão $q \vec{v} \times \vec{B}$ ou na expressão $I d \vec{l} \times \vec{B}$. Um outro produto vetorial (ou uma outra regra da mão direita) aparece na expressão matemática para calcular o campo magnético $\vec{B}$. Como temos este produto vetorial duplo na força magnética de Lorentz, ela não é tão problemática quanto o próprio conceito de campo magnético.

Por outro lado, as pessoas vão argumentar que o campo magnético é observado nas experiências que utilizam ímãs (como na famosa experiência de Oersted), ou então utilizando limalhas de ferro. Contudo, todas estas experiências podem ser explicadas pela força central de Ampère, que é uma lei de ação a distância, equação (4). As explicações para estas experiências utilizando a força de Ampère não utilizam o conceito de campos magnéticos circulando ao redor de um fio conduzindo uma corrente 
elétrica. Aliás, este aspecto sempre foi o ponto de vista defendido pelo próprio Ampère. Cito aqui sua obra mais importante, Teoria dos Fenômenos Eletrodinâmicos, Deduzida Unicamente da Experiência. Esta obra já está totalmente traduzida para a língua portuguesa ${ }^{21}$ :

A época marcada pelos trabalhos de Newton, na história das ciências, não é somente [o período] das descobertas mais importantes que o homem fez sobre as causas dos grandes fenômenos da natureza, é também a época na qual o espírito humano abriu uma nova rota nas ciências que tem por finalidade o estudo desses fenômenos.

Até então, tinham-se quase que exclusivamente procurado as causas [dos fenômenos naturais] no impulso de um fluido desconhecido, que arrastava as partículas materiais seguindo a direção de suas próprias partículas. E sempre que se via um movimento giratório, imaginava- se um turbilhão no mesmo sentido.

Newton nos ensinou que esse tipo de movimento, como todos os que a natureza nos oferece, deve ser reduzido pelo cálculo a forças agindo sempre entre duas partículas materiais, seguindo a reta que as une, de maneira que a ação exercida por uma delas sobre a outra seja igual e oposta à ação que esta última [partícula] exerce ao mesmo tempo sobre a primeira. Consequentemente, quando se supõem essas duas partículas invariavelmente ligadas entre si, disso não pode resultar qualquer movimento [do centro de massa do sistema] devido à ação mútua entre elas.

\section{$[\ldots]$}

Não parece que este caminho - o único que pode conduzir a resultados independentes de toda hipótese - seja preferido pelos físicos do resto da Europa, tal como o é [preferido] pelos franceses. E o sábio ilustre ${ }^{22}$ que viu pela primeira vez os polos de um ímã transladados pela ação de um fio condutor em direções perpendiculares à direção do fio concluiu que a matéria elétrica girava em torno dele, e empurrava os polos no sentido de seu movimento, precisamente como Descartes entendia que a matéria de seus turbilhões girava no sentido das revoluções planetárias. Guiado pelos princípios da filosofia newtoniana, reduzi o fenômeno observado pelo Sr. Oerstedt como se fez com respeito a todos aqueles do mesmo gênero que nos oferece a natureza - em termos de forças agindo sempre ao longo da reta que une as duas partículas, entre as quais as forças se exercem. [...]

\section{CONCLUSÃO}

Minha conclusão é a de que existem muitos aspectos positivos relacionados com a ação a distância. Entre eles posso citar a simplicidade deste enfoque, os resultados poderosos que são obtidos com esta suposição tanto no eletromagnetismo quanto na gravitação, a implementação matemática do princípio de Mach sobre a origem da inércia, o fato de que a primeira equação de onda descrevendo a propagação de perturbações eletromagnéticas foi obtida com a ação a distância (antes de Maxwell), etc. Existem também muitos problemas com a ação por contato baseada tanto no conceito de campos quanto em teorias balísticas (que supõem a emissão de partículas ou de corpúsculos pelos corpos macroscópicos que estão interagindo). Por estes motivos estou ficando a cada dia mais favorável em relação à ação a distância.

\section{AGRADECIMENTOS}

Este artigo é dedicado a Peter Graneau, o maior defensor da ação a distância que conheço. Aprendi bastante de muitos diálogos com ele ${ }^{23}$.

\footnotetext{
21 [Amp26], [Amp23], [Amp65], [Cha09], [AC11] e [AC15].

22 Ampère estava se referindo aqui a H. C. Ørsted.

23 [Ass14b].
} 


\section{REFERÊNCIAS}

[AC11] A. K. T. Assis and J. P. M. d. C. Chaib. Eletrodinâmica de Ampère: Análise do Significado e da Evolução da Força de Ampère, Juntamente com a Tradução Comentada de Sua Principal Obra sobre Eletrodinâmica. Editora da Unicamp, Campinas, 2011. ISBN: 9788526809383.

[AC15] A. K. T. Assis and J. P. M. C. Chaib. Ampère's Electrodynamics - Analysis of the Meaning and Evolution of Ampère's Force between Current Elements, together with a Complete Translation of His Masterpiece: Theory of Electrodynamic Phenomena, Uniquely Deduced from Experience. Apeiron, Montreal, 2015. Disponível em www.ifi.unicamp.br/ assis.

[AH07] A. K. T. Assis and J. A. Hernandes. The Electric Force of a Curent: Weber and the Surface Charges of Resistive Conductors Carrying Steady Currents. Apeiron, Montreal, 2007. Disponível em www.ifi.unicamp.br/ assis.

[AH09] A. K. T. Assis and J. A. Hernandes. A Força Elétrica de uma Corrente: Weber e as Cargas Superficiais de Condutores Resistivos com Correntes Constantes. Edusp e Edufal, São Paulo e Maceió, 2009. Volume 73 da Coleção Acadêmica. ISBNs: 9788531411236 e 9788571774315.

[AH13] A. K. T. Assis and J. A. Hernandes. Elektrischer Strom und Oberflächenladungen: was Wilhelm Weber schon vor mehr als 150 Jahre wusste. Apeiron, Montreal, 2013. Tradução de H. Härtel. Disponível em www.ifi.unicamp.br/ assis.

[Amp23] A.-M. Ampère. Mémoire sur la théorie mathématique des phénomenes électro-dynamiques uniquement déduite de l'expérience, dans lequel se trouvent réunis les Mémoires que M. Ampère a communiqués à l'Académie royale des Sciences, dans les séances des 4 et 26 décembre 1820, 10 juin 1822, 22 décembre 1823, 12 septembre et 21 novembre 1825. Mémoires de l'Académie Royale des Sciences de I'Institut de France, 6:175-387, 1823. Apesar da data, este volume foi publicado apenas em 1827.

[Amp26] A.-M. Ampère. Théorie des Phénomenes Électro-dynamiques, Uniquement Déduite de IExpérience. Méquignon-Marvis, Paris, 1826.

[Amp65] A.-M. Ampère. On the Mathematical Theory of Electrodynamic Phenomena, Experimentally Deduced. In R. A. R. Tricker, Early Electrodynamics - The First Law of Circulation, pages 155200, New York, 1965. Pergamon. Partial English translation by O. M. Blunn of Ampère's work "Mémoire sur la théorie mathématique des phénomenes électrodynamiques uniquement déduite de l'expérience", Mémoires de l'Académie royale des Sciences de I'Institut de France, Vol. 6, pp. 175-387 (1823), issued 1827.

[Ass89] A. K. T. Assis. On Mach's principle. Foundations of Physics Letters, 2:301-318, 1989.

[Ass93] A. K. T. Assis. Compliance of a Weber's force law for gravitation with Mach's principle. In P. N. Kropotkin et al., editor, Space and Time Problems in Modern Natural Science, Part II, pages 263-270, St.-Petersburg, 1993. Tomsk Scientific Center of the Russian Academy of Sciences. Series: "The Universe Investigation Problems," Issue 16.

[Ass94] A. K. T. Assis. Weber s Electrodynamics. Kluwer Academic Publishers, Dordrecht, 1994. ISBN: 0792331370.

[Ass95] A. K. T. Assis. Weber's law and Mach's principle. In J. B. Barbour and H. Pfister, editors, Mach's Principle - From Newton's Bucket to Quantum Gravity, pages 159-171, Boston, 1995. Birkhauser.

[Ass98] A. K. T. Assis. Mecânica Relacional. Editora do Centro de Lógica, Epistemologia e História da Ciência da UNICAMP/FAPESP, Campinas, 1998. Disponível em www.ifi.unicamp.br/ assis. 
[Ass99] A. K. T. Assis. Arguments in favour of action at a distance. In A. E. Chubykalo, V. Pope, and R. Smirnov-Rueda, editors, Instantaneous Action at a Distance in Modern Physics - "Pro" and "Contra", pages 45-56, Commack, 1999. Nova Science Publishers.

[Ass13] A. K. T. Assis. Mecânica Relacional e Implementação do Princípio de Mach com a Força de Weber Gravitacional. Apeiron, Montreal, 2013. Disponível em www.ifi.unicamp.br/ assis.

[Ass14a] A. K. T. Assis. Relational Mechanics and Implementation of Mach's Principle with Weber's Gravitational Force. Apeiron, Montreal, 2014. Disponível em www.ifi.unicamp.br/ assis.

[Ass14b] A. K. T. Assis. Text in memory of Peter Graneau (1921-2014). Infinite Energy, 114:14-15, 2014.

[Ass15] A. K. T. Assis. Eletrodinâmica de Weber: Teoria, Aplicações e Exercícios. Editora da Unicamp, Campinas, 2015. Segunda edição. e-ISBN: 978-85-268-1240-6.

[BA01] M. d. A. Bueno and A. K. T. Assis. Inductance and Force Calculations in Electrical Circuits. Nova Science Publishers, Huntington, New York, 2001. ISBN: 1560729171.

[BA15] M. Bueno and A. K. T. Assis. Cálculo de Indutância e de Força em Circuitos Elétricos. Apeiron, Montreal, 2015. $2^{a}$ edição. Disponível em: www.ifi.unicamp.br/ assis.

[Bro82] G. B. Brown. Retarded Action-at-a-Distance. Cortney Publications, Luton, 1982.

[Cha09] J. P. M. d. C. Chaib, 2009. Tese de Doutorado: "Análise do Significado e da Evolução do Conceito de Força de Ampère, juntamente com a Tradução Comentada de sua Principal Obra sobre Eletrodinâmica." Universidade Estadual de Campinas - UNICAMP (Campinas, SP). Orientador: A. K. T. Assis. Disponível em www.ifi.unicamp.br/ assis e www.repositorio.unicamp.br/handle/REPOSIP/262049.

[CSR96] A. E. Chubykalo and R. Smirnov-Rueda. Action at a distance as a full- value solution of Maxwell equations: the basis and application of the separated-potentials method. Physical Review E, 53:5373-5381, 1996.

[CSR97] A. E. Chubykalo and R. Smirnov-Rueda. Convection displacement current and generalized form of Maxwell-Lorentz equations. Modern Physics Letters A, 12:1-24, 1997.

[CSR98] A. E. Chubykalo and R. Smirnov-Rueda. Reply to "Comment on 'Action at a distance as a fullvalue solution of Maxwell equations: the basis and application of the separated-potentials method. Physical Re- view E, 57:1-4, 1998.

[GA94] P. Graneau and A. K. T. Assis. Kirchhoff on the motion of electricity in conductors. Apeiron, 19:19-25, 1994.

[GG93] P. Graneau and N. Graneau. Newton Versus Einstein - How Matter Interacts with Matter. Carlton Press, New York, 1993.

[Kir49] G. Kirchhoff. Ueber eine Ableitung der Ohm'schen Gesetze, welche sich an die Theorie der Elektrostatik anschliesst. Annalen der Physik, 78:506-513, 1849. Reimpresso em G. Kirchhoff's Gesammelte Abhandlungen (Barth, Leipzig, 1882), págs. 49-55.

[Kir50] G. Kirchhoff. On a deduction of Ohm's law in connexion with the theory of electrostatics. Philosophical Magazine, 37:463-468, 1850.

[Kir57a] G. Kirchhoff. On the motion of electricity in wires. Philosophical Magazine, 13:393-412, 1857.

[Kir57b] G. Kirchhoff. Ueber die Bewegung der Elektricitat in Drahten. Annalen der Physik, 100:193217, 1857. Reprinted in G. Kirchhoff's Gesammelte Abhandlungen (Barth, Leipzig, 1882), pp. 131-154. 
[Kir57c] G. Kirchhoff. Ueber die Bewegung der Elektricität in Leitern. Annalen der Physik, 102:529-544, 1857. Reprinted in G. Kirchhoff's Gesammelte Abhandlungen (Barth, Leipzig, 1882), pp. 154-168.

[Mac11] E. Mach. On the definition of mass. In History and Root of the Principle of the Conservation of Energy, pages 80-85. Open Court, Chicago, 1911. Translated from the German and annotated by P. E. B. Jourdain.

[Mac60] E. Mach. The Science of Mechanics - A Critical and Historical Account of Its Development. Open Court, La Salle, sixth edition,1960. Translated by J. McCormack.

[Mac14] E. Mach. História e Raízes do Princípio de Conservação de Energia. Eduerj, Rio de Janeiro, 2014. Tradução de G. D. Leitão. Revisão técnica de A. A. P. Videira.

[New79] I. Newton. Opticks. Dover, New York, 1979.

[New96] I. Newton. Óptica. Edusp, São Paulo, 1996. Tradução, introdução e notas de A. K. T. Assis. ISBN: 8531403405.

[P095] N. V. Pope and A. D. Osborne. Instantaneous gravitational and inertial action-at-a-distance. PhysicsEssays, 8:384-397, 1995.

[Pog57] J. C. Poggendorff. Bermerkung zu dem Aufsatz des Herrn Prof. Kirchhoff. Annalen der Physik, 100:351-352, 1857. Reprinted in W. Weber's Werke, Vol. 4, p. 242, H. Weber (ed.), (Springer, Berlin, 1894).

[Van97] T. Van Flandern. The speed of gravity - what the experiments say. Meta Research Bulletin, 6:49-62, 197.

[Web46] W. Weber. Elektrodynamische Maassbestimmungen - Über ein allgemeines Grundgesetz der elektrischen Wirkung. Abhandlungen bei Begründung der Königl. Sächs. Gesellschaft der Wissenschaften am Tage der zweihundertjährigen Geburtstagfeier Leibnizen's herausgegeben von der Fürstl. Jablonowskischen Gesellschaft (Leipzig), pages 211-378, 1846. Reprinted in Wilhelm Weber's Werke, Vol. 3, H. Weber (ed.), (Springer, Berlin, 1893), pp. 25-214.

[Web64] W. Weber. Elektrodynamische Maassbestimmungen insbesondere über elektrische Schwingungen. Abhandlungen der Königl. Sächs. Geselschaft der Wissenschaften, mathematisch-physische Klasse, 6:571-716, 1864. Reprinted in Wilhelm Weber's Werke, Vol. 4, H. Weber (ed.), (Springer, Berlin, 1894), pp. 105-241.

[Web07] W. Weber, 2007. Determinations of electrodynamic measure: concerning a universal law of electrical action, 21st Century Science \& Technology, traduzido para a língua inglesa por S. P. Johnson, editado por L. Hecht e A. K. T. Assis. Disponível desde março de 2007 em http://21sci-tech.com/translation.html e www.ifi.unicamp.br/ assis.

[Whi73] E. T. Whittaker. A History of the Theories of Aether and Electricity, volume 1: The Classical Theories. Humanities Press, New York, 1973.

[Whi91] C. K. Whitney. A gedanken experiment with relativistic fields. Galilean Electrodynamics, 2:28-29, 1991.

[Whi92] C. K. Whitney. What's wrong with standard relativistic fields? Galilean Electrodynamics, 3:8992, 1992. 